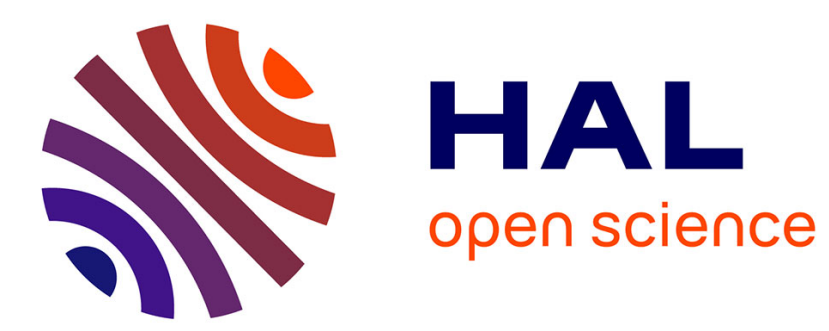

\title{
Evidence for a critical end point in [111]-stressed RbCaF3
}

\author{
J.Y. Buzaré, W. Berlinger, K.A. Müller
}

\section{To cite this version:}

J.Y. Buzaré, W. Berlinger, K.A. Müller. Evidence for a critical end point in [111]-stressed RbCaF3. Journal de Physique Lettres, 1985, 46 (5), pp.201-205. 10.1051/jphyslet:01985004605020100 jpa00232500

\section{HAL Id: jpa-00232500 https://hal.science/jpa-00232500}

Submitted on 1 Jan 1985

HAL is a multi-disciplinary open access archive for the deposit and dissemination of scientific research documents, whether they are published or not. The documents may come from teaching and research institutions in France or abroad, or from public or private research centers.
L'archive ouverte pluridisciplinaire HAL, est destinée au dépôt et à la diffusion de documents scientifiques de niveau recherche, publiés ou non, émanant des établissements d'enseignement et de recherche français ou étrangers, des laboratoires publics ou privés. 
Classification

Physics Abstracts

$64.60 \mathrm{~K}-64.70 \mathrm{~K}-76.30 \mathrm{~K}$

\title{
Evidence for a critical end point in [111]-stressed $\mathrm{RbCaF}_{3}$
}

\author{
J. Y. Buzaré (*), W. Berlinger and K. A. Müller \\ IBM Zurich Research Laboratory, 8803 Rüschlikon, Switzerland
}

(Reçu le 23 novembre 1984, accepté le 14 janvier 1985)

\begin{abstract}
Résumé. - Dans $\mathrm{RbCaF}_{3}$, pour une contrainte $\sigma>\sigma_{\mathrm{E}}$ appliquée suivant [111], une ligne de transitions de type Ising sépare une phase pseudo-cubique d'une phase trigonale. Elle rencontre sous un angle fini une ligne de transitions de premier ordre qui borde une phase pseudo-tétragonale. On localise ainsi un point critique terminal à $\sigma_{\mathrm{E}}=0,10 \pm 0,01 \mathrm{kbar}$ et $T_{\mathrm{E}}=196,3 \pm 0,1 \mathrm{~K}$. Le saut du paramètre d'ordre est monotone le long de la ligne de premier ordre. Pour établir le diagramme de phase on a utilisé la Résonance Paramagnétique électronique du centre $\mathrm{Gd}^{3+}-\mathrm{O}^{2-}$.

Abstract. - In $\mathrm{RbCaF}_{3}$, a [111] stress $\sigma>\sigma_{\mathrm{E}}$ induces an Ising line separating the pseudo-cubic from the trigonal phase which impinges at finite angle on a first-order boundary to the tetragonal phase. Thus, a critical end point (CEP) occurs sited at $\sigma_{\mathrm{E}}=0.10 \pm 0.01 \mathrm{kbar}$ and $T_{\mathrm{E}}=196.3 \pm$ $0.1 \mathrm{~K}$. The discontinuity jump of the order parameter along this boundary is monotonous across the CEP. The Paramagnetic Resonance of the $\mathrm{Gd}^{3+}-\mathrm{O}^{2-}$ centre was used as a symmetry probe to establish the phase diagram.
\end{abstract}

Multicritical points of various topologies have recently attracted considerable attention [1], the critical end points (CEP) especially attracting theoretical interest [2]. Blankschtein and Mukamel [3] have investigated theoretically structural phase transitions which are first-order cubic-to-tetragonal at zero stress. They found the phase diagram exhibited a CEP under [111] compressional stresses : a $q=3$ state Potts transition line which should be of first-order character, and an Ising line impinging on it at a finite stress. They predicted this phase diagram to be realizable in $\mathrm{BaTiO}_{3}, \mathrm{KMnF}_{3}$ and $\mathrm{RbCaF}_{3}$. The idea was tested in $\mathrm{KMnF}_{3}$ by specific-heat measurements [4]. Preliminary results were interpreted in this way, locating a CEP at $\sigma_{\mathrm{E}} \simeq 0.2 \mathrm{kbar}$ and $T_{\mathrm{E}} \simeq 187.35 \mathrm{~K}$, but the nature of the high-temperature transition has not been firmly established. In addition, the symmetry of the phases remained unknown. Furthermore, despite the transition at zero stress being continuous, EPR [5] and neutron-scattering [6] experiments indicated this kind of behaviour in [111]-stressed $\mathrm{SrTiO}_{3}$. But the CEP is not sufficiently resolved in as much as the angle under which the Ising line joins the Potts line is concerned.

In this Letter, we present conclusive EPR investigations of the phase diagram for [111]-stressed $\mathrm{RbCaF}_{3}$ confirming the theory. The EPR technique is known to provide accurate measurements of components of the order parameter, and clear determination of the phase symmetries.

$\left({ }^{*}\right)$ Permanent address : Laboratoire de Spectroscopie du Solide (E.R.A. CNRS, $\mathbf{n}^{\circ}$ 682), 72017 Le Mans Cedex, France. 
$\mathrm{RbCaF}_{3}$ is a compound with perovskite structure undergoing a slightly first-order phase transition [7] from the cubic $\mathrm{O}_{\mathrm{h}}^{1}$ to the tetragonal $\mathrm{D}_{4 \mathrm{~h}}^{18}$ space group. The order parameter of the transition is the rotational angle of the $\mathrm{CaF}_{6}$ octahedra $\varphi$ which has three components $\varphi_{[100]}$, $\varphi_{[010]}$ and $\varphi_{[001]}$. This transition should be continuous according to mean-field theory and may be explained to be driven first-order by critical fluctuations : the strongly anisotropic soft-mode behaviour is connected with nearly two-dimensional correlated (100), (010) and (001) octahedral sheets which tend to keep the crystal cubic [8]. However, the crossover from first-order to continuous transition through a tricritical point (TCP) could be induced by symmetry-breaking fields of near [001] stress $[9,10]$ : the TCP is believed to be of Lifshitz type owing to the strongly quartic character of the soft phonon dispersion [11].

$\mathrm{RbCaF}_{3}$ crystals doped with $\mathrm{Gd}_{2} \mathrm{O}_{3}$ were grown by one of us (J. Y. B.) by the Bridgman-Stockbarger method. In this experiment, a compressional stress $\sigma$ was applied along a [111] cubic direction. Therefore, the sample is a cylinder machined to a diameter of $2.9 \mathrm{~mm}$ and a height of $4.7 \mathrm{~mm}$ with carefully polished parallel (111) end planes. The EPR experiments were carried out at microwave $\mathrm{K}$-band in the IBM multipurpose cavity where the stress is applied with a stainlesssteel hollow force transmitting tube and the various accessories as previously described; the sample temperature is kept constant to better than $10^{-4} \mathrm{~K}$ [12]. At room temperature, the sample shows two different EPR spectra : one results from $\mathrm{Gd}^{3+}$ ions on $\mathrm{Ca}^{2+}$ sites, the other from $\mathrm{Gd}^{3+}-\mathrm{O}^{2-}$ electrically neutral pairs substituted to $\mathrm{Ca}^{2+}-\mathrm{F}^{-}$bonds. The geometry of the apparatus allows application of the stress perpendicular to the external magnetic field $\mathbf{H}$. In such a case, the EPR spectrum of $\mathrm{Gd}^{3+}$ is insensitive to the octahedra rotations around [111], whereas the $\mathrm{Gd}^{3+}-\mathrm{O}^{2-}$ pair is sensitive thereto. The spectrum exhibits strong axial lines corresponding to a large quadrupolar parameter $b_{2}^{0}$ in the spin Hamiltonian. This yields a strong dependence of the line positions on the angle between the magnetic field and the pair axis [7]. In the cubic phase for $\mathbf{H}$ along [110], the lines owing to pairs pointing along [100] and [010] coincide. In the tetragonal phase, octahedral rotations by $\pm \varphi$ around [001] result in a splitting of these lines proportional to $\varphi$, whereas rotations around [100] and [010] leave the lines at their cubic-phase positions. We can then infer that when the magnetic field is along a twofold cubic axis, for any octahedral rotational vector, the line splitting is induced by this vector component which lies along the fourfold cubic axis perpendicular to the magnetic field.

Throughout this work, owing to our particular geometry, $\mathbf{H}$ is kept along [110]. Then the [001] components of the octahedral rotational vectors are monitored. We concentrate on the EPR line occurring at $H \simeq 3500 \mathrm{G}$ in the cubic phase : its splitting resulting from octahedral rotations is given by $\Delta H=B \varphi_{[001]}$ with $B=(125 \pm 1) \mathrm{G} /$ degree.

At zero stress, the well-known slightly first-order tetragonal-to-cubic transition is observed at $T_{\mathrm{c}}=196.05 \pm 0.10 \mathrm{~K}$ for this newly grown crystal. Application of [111] stress at sufficiently low temperature leaves the tetragonal phase almost unchanged. An EPR spectrum is shown in figure 1a for $\sigma_{[111]}=0.31 \mathrm{kbar}$ corresponding to the highest force $(20 \mathrm{~kg})$ applied on the crystal. The only difference from the stress-free spectrum concerns the inner lines. The observed splitting which increases on heating, gives evidence for removal of the tetragonal symmetry : the rotational vectors $\varphi$ move slightly away from the fourfold axes towards [111] in each domain. Then the $\mathrm{Gd}^{3+}-\mathrm{O}^{2-}$ pairs rotated by $\pm|\varphi|$ around near [100] and [010] directions become inequivalent. This splitting $\Delta H_{\mathrm{i}}$ is proportional to the $\varphi_{\mathrm{i}[001]}$ component figures $1 \mathrm{a}$ and $1 \mathrm{e}$. The two outer lines result from pairs located in domains with near [001] order-parameter direction. Their splitting $\Delta H_{\mathrm{o}}$ is proportional to the $\varphi_{\mathrm{o}[001]}$ component. The angle $\alpha$ by which the $\varphi$ 's are rotated away from the fourfold axes may be estimated from $\operatorname{tg} \alpha=\sqrt{2} \varphi_{\mathrm{i}[001]} / \varphi_{\mathrm{o}[001]}=\sqrt{2} \Delta H_{\mathrm{i}} / \Delta H_{\mathrm{o}}$. This leads to $\alpha \simeq 16.4^{\circ}$ at $T=196.35 \mathrm{~K}$ and $8.7^{\circ}$ at $195 \mathrm{~K}$ for $\sigma_{[111]}=0.31 \mathrm{kbar}$. At a critical temperature $T_{\mathrm{p}}\left(T_{\mathrm{p}}=196.45 \mathrm{~K}\right.$ for this stress), the spectrum changes discontinuously to figure $1 \mathrm{~b}$. The lines observed above and below this critical temperature coexist. This has to be seen as evidence for a first-order transition. Above $T_{\mathrm{p}}$, two lines are recorded (Fig. 1c). They result from alternate 

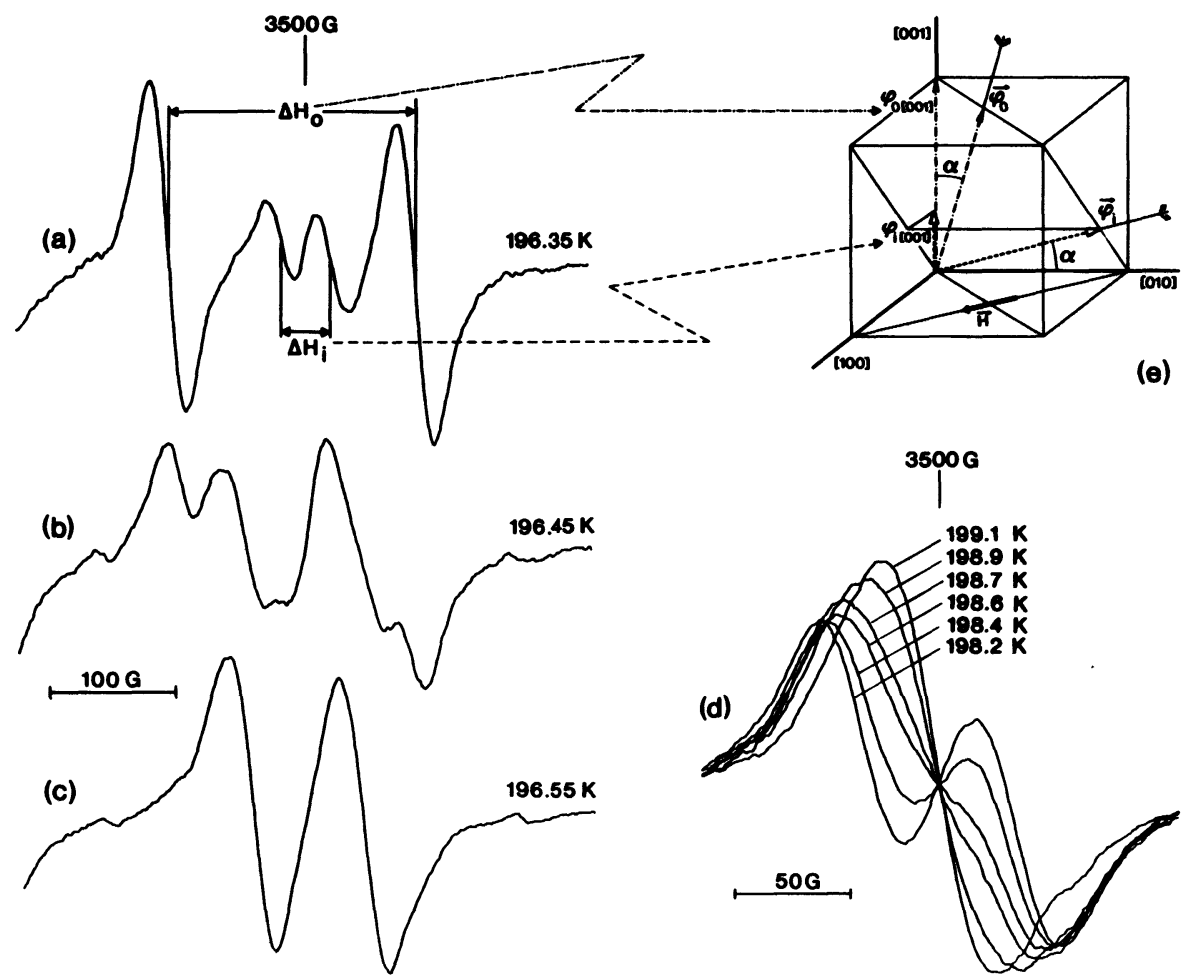

Fig. 1. - (a), (b) and (c) : EPR line evolution at the first-order transition $\left(T_{\mathrm{p}}\right)$. (a) $T \simeq T_{\mathrm{p}}$, tetragonal phase (including explanations of the splittings $\Delta H_{\mathrm{o}}$ and $\Delta H_{\mathrm{i}}$ );(b) $T \simeq T_{\mathrm{p}}$, tetragonal and trigonal phase coexistence;(c) $T \gtrsim T_{\mathrm{p}}$, trigonal phase. (d) EPR line evolution at the second-order transition $\left(T_{\mathrm{l}}\right)$. (e) Geometry of rotational order-parameter vectors and their [001] projection in the pseudo-tetragonal phase.

rotations of the octahedra around the [111] direction parallel to the stress. Thus, we obtain a monodomain $\mathrm{RbCaF}_{3}$ sample with $\mathrm{R} \overline{3} \mathrm{c}$ structure. The splitting between the two lines is proportional to the order parameter $\varphi_{[111]}$ in this $\mathrm{R} \overline{3} \mathrm{c}$ phase. It decreases continuously down to zero when $T$ is raised up to $T_{\mathrm{I}}=(198.95 \pm 0.10) \mathrm{K}$ (Fig. 1d). For $T \geqslant T_{\mathrm{I}}$, only one line is observed. It results from $\mathrm{Gd}^{3+}-\mathrm{O}^{2-}$ pairs along [100] and [010] which are equivalent in the pseudo-cubic phase. Therefore, $T_{p}$ is the temperature at which the jump from the tetragonal to the trigonal phase occurs. Determination of $T_{\mathrm{I}}$ is not so straightforward owing to overlapping and broadening of the EPR lines as the trigonal-cubic transition is approached. We locate it for the temperature at which an inflection point is observed in the variation of the peak-to-peak distance of the EPR line versus temperature after the splitting has become unresolved.

The temperature dependence of the spectra was analysed for a large variety of applied stresses. Our results are summarized in figure 2 where the phase diagram is plotted. From the data, it may be seen that the slightly first-order cubic-tetragonal phase transition extends to $\sigma_{\mathrm{E}}=(0.10 \pm$ $0.01) \mathrm{kbar}$. In this stress range $\left(\sigma<\sigma_{\mathrm{E}}\right)$, the transition temperature $T_{\mathrm{c}}$ is but slightly modified by the applied stress $\left(\mathrm{d} T_{\mathrm{c}} / \mathrm{d} \sigma_{[111]} \gtrsim 0\right)$. Furthermore, the order-parameter discontinuity at $T_{\mathrm{c}}$ remains constant within the experimental error (Fig. 3). Thus, these small compressional [111]applied stresses do not alter the octahedral sheet correlations. For $\sigma>\sigma_{\mathrm{E}}$, a trigonal phase arises and we have two phase boundaries. At low temperature, a first-order line $\left(T_{\mathrm{p}}\right)$ separates the pseudo-tetragonal from the trigonal phase, merging the $T_{\mathrm{c}}$ line. Note that $T_{\mathrm{p}}$ is essentially stress independent. Along the $T_{\mathrm{p}}$ line, no change of the $\varphi_{\mathrm{o}[001]}$ order-parameter component is 


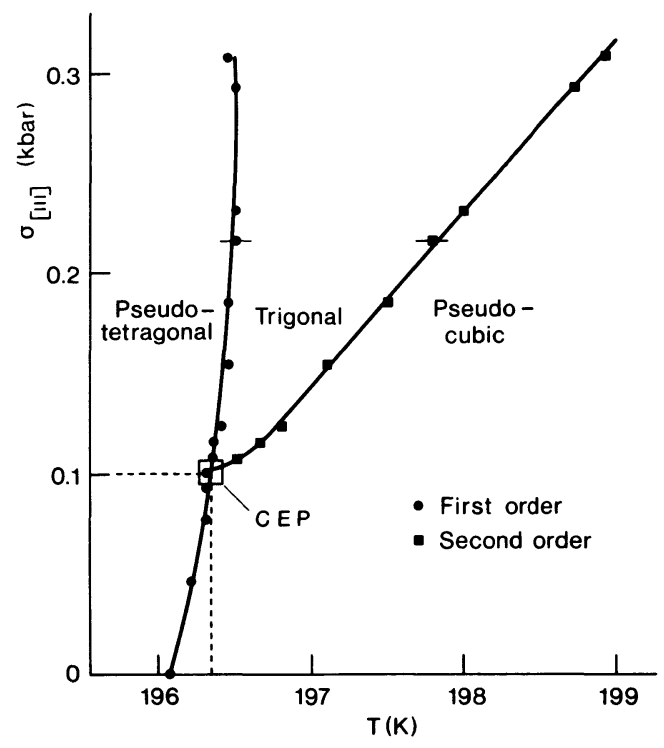

Fig. 2. - Phase diagram $(\sigma, T)$ of [111]-stressed $\operatorname{RbCaF}_{3}$.
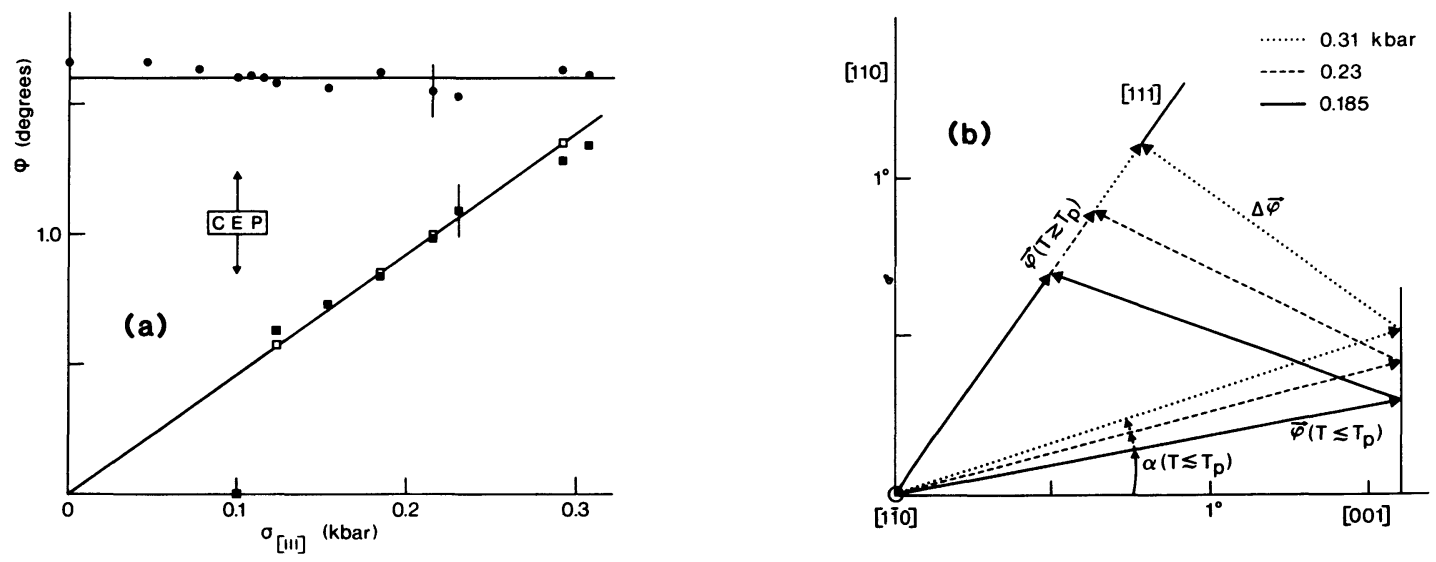

Fig. 3. - (a) Component $\varphi_{\mathrm{o}[001]}$ of the order parameter in the tetragonal phase at $T \lesssim T_{\mathrm{c}}$ or $T_{\mathrm{p}}$ deduced from $\Delta H_{0}(\bullet) ; \varphi_{[111]}$ at $T \gtrsim T_{\mathrm{p}}$ deduced from the line splitting $(\boldsymbol{\square})$ and measured by rotating $\mathbf{H}$ in the (111) plane ( $\square$ ) versus $\sigma_{[111]}$ (b) Order-parameter evolution at the $T_{p}$ transition for three applied stresses.

observed (Fig. 3a) whereas the $\varphi_{\text {i[001] }}$ increases : therefore $|\boldsymbol{\varphi}|$ and $\alpha$ increase with the applied stress, while the order-parameter discontinuity $\Delta \varphi$ and the $\varphi$ direction jump from near fourfold cubic axes towards [111] decrease. In figure 3a, the variations of $\varphi_{[111]}$ versus $\sigma_{[111]}$ along the $T_{p}$ line are also plotted. Except for the neighbourhood of $\sigma_{\mathrm{E}}$, the experimental points lie on a straight line which seems to pass through the origin with a slope equal to $4.6^{\circ} / \mathrm{kbar}$. These results for some applied stresses are summarized in figure $3 \mathrm{~b}$. They corroborate the first-order character of the transition. The second boundary is a second-order line between the trigonal and the cubic phases. It meets the former line at $\sigma_{\mathrm{E}}=0.10 \pm 0.01 \mathrm{kbar}$, and $T_{\mathrm{E}}=196.3 \pm 0.1 \mathrm{~K}$ at a finite angle 
thus locating a multicritical point. At this multicritical point, a continuous first-order line meets a second-order line $T_{\mathrm{I}}$ : this is a CEP. Except close to this point, $T_{\mathrm{I}}$ varies linearly with $\sigma_{\text {[111] }}$ according to $\mathrm{d} T_{\mathrm{I}} / \mathrm{d} \sigma_{[111]}=(11.8 \pm 0.1) \mathrm{K} /$ bar.

Qualitatively, the gross features of this phase diagram may be quite easily understood. They arise from the competition between ordering along [100] directions favoured at zero stress and ordering along [111] favoured by the uniaxial stress $\sigma_{[111]}$. For convenience, in the following discussion let us choose the components of $\varphi$ as $\varphi_{1}$ along [111] and $\varphi_{2}$ and $\varphi_{3}$ in the (111) plane. As the temperature is lowered at constant $\sigma>\sigma_{\mathrm{E}}, \varphi_{1}$ orders first through a continuous Isinglike $(n=1)$ transition : the stress wins. Lowering the temperature further, $\varphi_{2}$ and $\varphi_{3}$ also order at a first-order transition : the natural ordering wins. Furthermore, owing to the threefold symmetry of the ordering in the (111) plane for $T<T_{\mathrm{p}}$ this transition may be seen as three-state Potts-like [13]. For stresses $\sigma<\sigma_{\mathrm{E}}$, the disappearance of the trigonal phase indicates that the applied stresses are definitely too small to compete with the "natural » ordering.

In conclusion, one can state that these compressional [111]-stress EPR results in $\mathrm{RbCaF}_{3}$ corroborate the topology of the predicted phase diagram and its CEP [3, 14]. It is similar to the diagram in $\mathrm{KMnF}_{3}$ [4], but in the $\mathrm{RbCaF}_{3}$ EPR experiments the amount and direction of the order parameter in the tetragonal and trigonal phases has been measured and awaits quantitative theoretical computation.

\section{Acknowledgments.}

One of us (J. Y. B.) would like to acknowledge the hospitality of the IBM Zurich Research Laboratory where he was visiting when this work was performed.

\section{References}

[1] See : Proc. NATO Adv. Study Inst. Multicritical Phenomena, Geilo 1983, R. Pynn and A. Skjeltorp eds. (Plenum, New York) 1984.

[2] Kerszberg, M. and Mukamel, D., Phys. Rev. Lett. 43 (1979) 293 and Phys. Rev. B 23 (1981) 3943 and 3953.

[3] Blankschtein, D. and Mukamel, D., Phys. Rev. B 25 (1982) 6939.

[4] Fossheim, K., in [1].

[5] Mueller, K. A., Berlinger, W. and Slonczewski, J. C., Phys. Rev. Lett. 25 (1970) 734 ;

Mueller, K. A. and Berlinger, W., Z. Phys. B 46 (1982) 81.

[6] Currat, R., Mueller, K. A., Berlinger, W. and Denoyer, F., Phys. Rev. B 17 (1978) 2937.

[7] Buzaré, J. Y., Rousseau, J. J. and Fayet, J. C., J. Physique Lett. 38 (1977) L-445 ;

Buzaré, J. Y., Fayet-Bonnel, M. and Fayet, J. C., J. Phys. C 13 (1980) 857 ;

Buzaré, J. Y., Fayet-Bonnel, M. and Fayet, J. C., J. Phys. C 14 (1981) 67.

[8] Nattermann, T., J. Phys. C 9 (1976) 3337.

[9] Aharony, A. and Bruce, A. D., Phys. Rev. Lett. 42 (1979) 462.

[10] Buzaré, J. Y., Fayet, J. C., Berlinger, W. and Mueller, K. A., Phys. Rev. Lett. 42 (1979) 465.

[11] Rousseau, M., Nouet, J. and Almairac, R., J. Physique 38 (1977) 1423.

[12] Berlinger, W. and Mueller, K. A., Rev. Sci. Instrum. 48 (1977) 1161.

BERLINGER, W., Magn. Res. Rev. (1984) in press.

[13] Aharony, A., Mueller, K. A. and Berlinger, W., Phys. Rev. Lett. 38 (1977) 33.

[14] Note the phase diagram shown in figure $3 \mathrm{~b}$ of [3] is schematic and therefore only the general topology is relevant. 